Journal of the Electrochemical Society, 1989, Volume 136, Issue 4, Pages 1243-1244.

ISSN: 0013-4651

DOI: $10.1149 / 1.2096864$

http://www.electrochem.org/

http://scitation.aip.org/getpdf/servlet/GetPDFServlet?filetype=pdf\&id=JESOAN000136000004001243000001\&idty pe $=$ cvips\&prog $=$ normal

(C) The Electrochemical Society, Inc.1989. All rights reserved. Except as provided under U.S. copyright law, this work may not be reproduced, resold, distributed, or modified without the express permission of The Electrochemical Society (ECS). The archival version of this work was published in Journal of the Electrochemical Society, Vol. 136, No. 4, 1989, pp. 1243-1244.

\title{
Pitting of Sputtered Aluminum Alloy Thin Films
}

\author{
G. S. Frankel, M. A. Russak, C. V. Jahnes, M. Mirzamaani,* and V. A. Brusic* \\ IBM Research Division, T.J. Watson Research Center, Yorktown Heights, New York. \\ *Electrochemical Society Active Member.
}

\section{INTRODUCTION}

Metallic films with thickness on the order of hundreds or thousands of angstroms are extensively used in the electronics industry. Due to obvious dimensional limitations, such thin films may be very susceptible to performance degradation resulting from corrosion. In this study, pitting of $\mathrm{Al}$ alloy thin films has been investigated. An advantage to studying thin films is that it is possible to produce alloys by thin film deposition techniques that are not easily produced by conventional metallurgical methods. Recent reports have described the exceptional pitting and corrosion resistance of thin film aluminum alloys (1-3). These alloys were metastable solid solutions produced by sputter deposition containing up to 15 atomic \% solute which is far in excess of the equilibrium solubility. In this study, we have taken a similar approach to look at a wide compositional range of several $\mathrm{Al}$ alloys.

\section{EXPERIMENTAL}

$\mathrm{Al}$ and Al binary alloy films approximately $2000 \AA$ thick were produced by DC magnetron co-sputter deposition on quartz wafers at room temperature. This is in contrast to the approach of Moshier et al. (1,2) who deposited with the substrate held at liquid nitrogen temperatures, a precaution which is apparently not necessary. Two $5 \mathrm{~cm}$ diameter magnetron sources, one with a pure Al target (99.99\%) and one with the desired solute element (Mo, Ta, $\mathrm{Nb}, \mathrm{Ti}$, or $\mathrm{Nb}$ ) were arranged so that several substrate wafers could be placed on an underlying platform along the symmetry line. In this manner, a range of compositions was deposited in one run on the substrates and, in fact, a slight composition gradient existed on each substrate. The composition of films on carbon substrates that had been placed between the quartz sample substrates were determined by Rutherford Backscattering Analysis. The composition of the film deposited on a given quartz substrate was estimated to be the average of the values of the carbon substrate samples to either side. The accuracy of this measurement is about $\pm 5 \%$. Due to this and the composition gradient that existed on each sample, there was some scatter of the pitting potential data. Multiple experiments were made for each composition and the scatter of the reported pitting potential values in some cases was quite good but at worst was about $\pm 100 \mathrm{mV}$. 
After metal deposition, a layer of mixed oxide dielectric was reactively sputtered using physical masks to define exposed metal areas of $0.495 \mathrm{~cm}^{2}$. This technique, described in detail elsewhere (4), was very successful at minimizing crevice attack.

The samples were tested in $0.1 \mathrm{M} \mathrm{NaCl}$ adjusted to $\mathrm{pH} 10$ with $\mathrm{NaOH}$. The solution was deaerated for at least $2 \mathrm{~h}$ prior to and during testing by bubbling with purified $\mathrm{N}_{2}$. The samples were, immersed for $15 \mathrm{~min}$ at open circuit and pitting potentials were then measured by scanning potentiodynamically from open circuit against an SCE reference electrode at $0.2 \mathrm{mV} / \mathrm{s}$ until breakdown. The specimens were observed during testing with a microprobe camera at $100 \mathrm{X}$ and the images were recorded on videotape. It was therefore possible to observe the growth of the pits to be certain that the attack was away from the dielectric masking layer so that the breakdown potential was indicative of pitting and not crevice attack.

\section{RESULTS}

The pitting or pit nucleation potentials, $\mathrm{E}_{\mathrm{np}}$, of pure $\mathrm{Al}$ and a number of $\mathrm{Al}$ binary alloy films are shown in Figure 1. The pitting potential of unalloyed Al thin films was $-690 \mathrm{mV} \mathrm{SCE}$, identical to values reported in the literature for bulk Al in $0.1 \mathrm{M} \mathrm{Cl}^{-}$(5). The addition of only about 5 atomic $\%$ of $\mathrm{Mo}, \mathrm{Nb}, \mathrm{Ta}, \mathrm{Ti}$, or $\mathrm{Cr}$ was sufficient to have a large effect on the pitting potential, increasing it to the range of -200 to $0 \mathrm{mV} \mathrm{SCE}$. The Al alloy thin films produced and studied in this work contained up to about 40 atomic \% solute. At high solute concentrations the Al alloy films had exceptionally high pitting potentials that exceed even that of AISI 304 stainless steel. The highest pitting potential for a given concentration was exhibited by the AlMo system. For the Al-Nb and Al-Ta alloys, the values of $\mathrm{E}_{\mathrm{np}}$ were somewhat lower than those for Al-Mo but all three increased monotonically with concentration. Al-Ta alloys with about 41 $\%$ Ta behave like pure Ta in that pitting was not observed up to $+1600 \mathrm{mV}$ SCE.

Al-Ti and Al-Cr alloys both exhibited a plateau at low concentrations during which the pitting potential was relatively low and independent of solute concentration. When the concentration of these alloys exceeded some critical value, the pitting potential then increased with increasing concentration. The critical concentration for $\mathrm{Al}-\mathrm{Cr}$ was about $35 \%$, about twice that of Al-Ti.

\section{SUMMARY}

A number of $\mathrm{Al}$ binary alloy thin films were studied over a range of composition up to $40 \%$ solute using a co-sputtering technique with the substrates at room temperature. These $\mathrm{Al}$ alloy films were tested in $0.1 \mathrm{M} \mathrm{NaCl}$ and found to have extraordinary pitting resistance.

\section{REFERENCES}

1. W. C. Moshier, G. D. Davis, J. S. Ahearn, and H. F. Hough, This Journal. 134, 2677 (1987).

2. W. C. Moshier, G. D. Davis, G. O. Cote, H. F. Hough, and M. E. Vogelsang, Martin Marietta Corp., Baltimore, MD, Annual Report to ONR under contract no. N00014-85-C-0638, September 1987.

3. H. Yoshioka, A. Kawashima, K. Asami, and K. Hashimoto, Abs. 219, p. 318, The Electrochemical Society, Extended Abstracts Vol. 87-2, Honolulu, HI, October 18-23, 1987.

4. G. S. Frankel, C. V. Jahnes, and M. A. Russak, accepted for publication in Corrosion. 


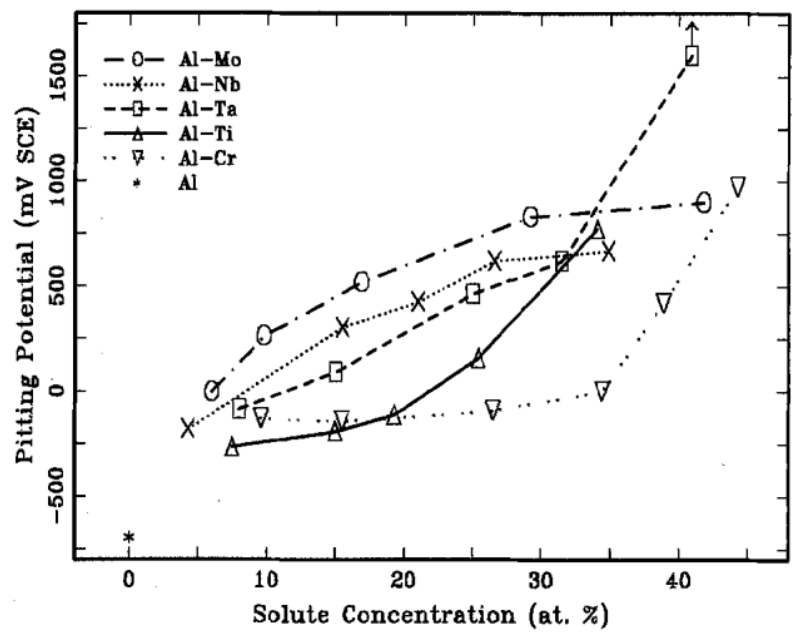

Figure 1. Pitting potentials of various $\mathrm{Al}$ alloy thin films in deaerated $\mathrm{pH} 100.1 \mathrm{M} \mathrm{NaCl}$ as a function of concentration. 